\title{
The unique carrier mobility of Janus MoSSe/GaN heterostructures
}

\author{
Wen-Jin Yin, Xiao-Long Zeng, Bo Wen, Qing-Xia Ge, Ying Xu, \\ Gilberto Teobaldi and Li-Min Liu
}

\section{Published version information}

Citation: W Yin et al. 'The unique carrier mobility of Janus MoSSe/GaN heterostructures.' Frontiers of Physics, vol. 16, no. 3 (2020): 33501.

DOI: $\underline{10.1007 / s 11467-020-1021-1}$

This is a post peer-review, pre-copyedit version of an article published in Frontiers of Physics. The final authenticated version is available online at the DOI above.

This version is made available in accordance with publisher policies. Please cite only the published version using the reference above. This is the citation assigned by the publisher at the time of issuing the AAM. Please check the publisher's website for any updates. 


\title{
The Unique Carrier Mobility of Janus MoSSe/GaN Heterostructures
}

Wen-Jin Yin ${ }^{l}$, Xiao-Long Zeng ${ }^{l}$, Bo Wen ${ }^{2}$, Qing-Xia Ge ${ }^{1}$, Ying Xu ${ }^{l}$, Gilberto Teobaldi ${ }^{3,4,5}$, and LiMin Liü ${ }^{* 2}$

${ }^{I}$ School of Physics and Electronic Science, Hunan University of Science and Technology, Xiangtan 411201, China

${ }^{2}$ School of Physics, Beihang University, Beijing 100083, China

${ }^{3}$ Scientific Computing Department, STFC UKRI, Rutherford Appleton Laboratory, Harwell Campus, OX11 0QX Didcot, United Kingdom

${ }^{4}$ Stephenson Institute for Renewable Energy, Department of Chemistry, University of Liverpool, L69 3BX Liverpool, United Kingdom

${ }^{5}$ School of Chemistry, University of Southampton, Highfield, SO17 1BJ Southampton, United Kingdom

Corresponding authors: liminliu@buaa.edu.cn

\begin{abstract}
Heterostructure is an effective approach in modulating the physical and chemical behavior of materials. Here, the first-principles calculations were carried out to explore the structural, electronic, and carrier mobility properties of Janus MoSSe/GaN heterostructures. This heterostructure exhibits a superior high carrier mobility of 281.28 $\mathrm{cm}^{2} \mathrm{~V}^{-1} \mathrm{~s}^{-1}$ for electron carrier and $3951.2 \mathrm{~cm}^{2} \mathrm{~V}^{-1} \mathrm{~s}^{-1}$ for hole carrier. Particularly, the magnitude of the carrier mobility can be further tuned by Janus structure and stacking modes for the heterostructure. It is revealed that the equivalent mass and elastic moduli strongly affect the carrier mobility of the heterostructure, while the deformation potential contribute the different carrier mobility for electron and hole of the heterostructure. These results suggested that the Janus MoSSe/GaN heterostructure has many potential applications for the unique carrier mobility.
\end{abstract}

Keywords: Janus Heterostructure; Carrier Mobility; First-principles Calculation

\section{Introduction}

The increasing development of technology triggers the revolution of electronic device or vehicle toward microminiaturization and multifunctional. ${ }^{[1-3]}$ It is well known that size and intrinsic property of a material are the two crucial factors. That means it should be in nanocrystalline, as well as own with desirable band gap and carrier mobility. The successful exfoliation of graphene shines the light on the potential of low dimensional material production. Meanwhile, it shows that graphene exhibits halfinteger quantum hall effect, high migration rate, and mass less carrier transport properties; ${ }^{[4]}$ However, the absence of band gap in pristine sheet leads to an extremely low on/off ratio, which severely limits its further application in nanoscale electronic device. ${ }^{[4-6]}$ Thus, it has aroused extensive attention to modulate the electronic structure.

Structure tailoring is a common method to control the electronic property, such as forming armchair or zigzag edge nanoribbons. Although a small band gap can be open, the carrier mobility would be dramatically reduced due to the missing of Dirac cone and scattering effect in the nanoribbons. ${ }^{[7,8]}$ For example, sub-10 nm nanoribbon field effect transistors shows that the carrier mobility dropped to less than $200 \mathrm{~cm}^{2} \mathrm{~V}^{-1} \mathrm{~s}^{-1}$ with 
diminishing width when the enough band gap opened. ${ }^{[9]}$ On the other hand, introducing defect, doping, or surface modification can also regulate the electronic property of a material. But it is totally unavoidable to bring foreign atom into the system, where it will often serve as an electron or hole carrier trap site.

Apart from above approaches, searching for graphene analogue is fascinating, and shows strong vitality. ${ }^{[10-12]}$ Transition metal dichalcogenides (TMDs) have received great interests. Through varying the collocation of chalcogenide S, Se, and Te atoms and transition metal Mo, W, Nb and $\mathrm{V}$ atoms, plenty of TMDs structures with tunable band gaps can be obtained. ${ }^{[13,14]}$ Single layer $\mathrm{MoS}_{2}$, as a typical member of TMDs family, is a semiconductor with a band gap of about $1.9 \mathrm{eV},{ }^{[15]}$ and it has been regarded as a promising candidate for field effect transistor with an on/off ratio exceeding $10^{8}{ }^{[16]}$ However, the previous result clearly shows that carrier mobility of a suspended $\mathrm{MoS}_{2}$ sheet is found to be in the range of $0.5-3 \mathrm{~cm}^{2} \mathrm{~V}^{-1} \mathrm{~s}^{-1}$, thus this small mobility will cause low efficiency for the electronic devices. ${ }^{[17-19]}$ Interestingly, Guo et al. found sorts of new metal oxides or sulfurs owing ultrahigh carrier mobility, together with superior oxidation resistance. ${ }^{[20,21]}$ Further work showed that through removing absorbates or depositing atop a high-dielectric layer, extrinsic scatters can be partially suppressed, and the value of carrier mobility can be increased to $200 \mathrm{~cm}^{2} \mathrm{~V}^{-1} \mathrm{~s}^{-1} \cdot{ }^{[22-25]}$ In addition, the magnitude of carrier mobility can also be remained in its armchair nanoribbons. ${ }^{[26]}$

Recently, Lu et al. designed out a new kind of Janus TMDs MoSSe, ${ }^{[27]}$ which is obtained through breaking the out-of-plane structural symmetry of $\mathrm{MoS}_{2} .{ }^{[28,29]}$ Superior to the $\mathrm{MoS}_{2}$, the Janus TMDs reported that an intrinsic dipole as well as large piezoelectricity could exist in the vertical direction of the Janus sheet. ${ }^{[30-33]}$ Furthermore, theoretical result shows that the carrier mobility of single layer is about $157 \mathrm{~cm}^{2} \mathrm{~V}^{-1} \mathrm{~s}^{-1}$ for hole carrier and $74 \mathrm{~cm}^{2} \mathrm{~V}^{-1} \mathrm{~s}^{-1}$ for electron carrier. The carrier mobility can be further adjusted by varying the thickness like forming double or triple layers as homo heterostructure with the electron carrier at $1194 \mathrm{~cm}^{2} \mathrm{~V}^{-1} \mathrm{~s}^{-1}$ and hole carrier at 5894 $\mathrm{cm}^{2} \mathrm{~V}^{-1} \mathrm{~s}^{-1}$ predicted by theoretical calculations. ${ }^{[30]}$ Inspired by this, it is interesting to know the effect of heterostructure. ${ }^{[34]}$ Because heterostructure can not only preserve the property of its individual component, but also introduce advanced functional. ${ }^{[35-37]}$ For example, the heterostructure of the different semiconductors could exhibit unusual band gap. ${ }^{[38,39]}$ What's more, intrinsic electric filed may generally exist in the heterostructure, and it would help to separate the carriers. ${ }^{[40-42]}$ Meanwhile, Schottky barrier in the heterostructure $^{[43]}$ can also effectively reduce the recombination of electron-hole pairs and improve the life time of the carriers. ${ }^{[44]}$ The formation of heterostructure requires the two components, owing some familiar behaviors. Luckily, the recent advancements in 2D group III-V compounds with simple wurtzite or zinc-blende structures like nitride GaN brings hope to further improve the single layer TMDs. The electronic band gap of single layer GaN can vary from $1.9 \mathrm{eV}$ to $3.0 \mathrm{eV}$ for different configurations. ${ }^{[45]}$ The lattice parameters of GaN are quite similar to single layer TMDs. ${ }^{[46]}$ And it has been reported that the formation of heterostructure with internal polarization can effectively reduce the band gap. ${ }^{[47]}$ Thus, it is interesting to know the carrier behavior of the heterostructure between single layer Janus TMDs and nitride GaN.

In this work, we aim to unveil the structural and electronic properties of the Janus 
$\mathrm{MoSSe} / \mathrm{GaN}$ heterostructure by the first-principles calculations. The outline of the paper can be organized as the following: Firstly, we systematically study the stabilities of the $\mathrm{MoSSe} / \mathrm{GaN}$ heterostructures. Then, we examine the carrier mobility and the electronic behavior of the heterostructures. At last, the crucial factors on determining carrier mobility behavior will be discussed.

\section{Computational Method}

All simulations were carried out at Density Functional Theory (DFT) level as implemented in the Vienna ab initio simulation program package (VASP), ${ }^{[48,49]}$ with the Perdew-Burke-Ernzerhof (PBE) exchange-correlation functional under the generalized gradient approximation (GGA). ${ }^{[50,51]}$ The projector augmented wave (PAW) method was used to represent the electronic density at the atomic cores. The plane-wave energy cutoff was $500 \mathrm{eV}$ and the converged energy is less than $1 \mathrm{meV}$ per atom, checked to be sufficiently accurate against calculations with higher cutoffs. The convergence criterion for the self-consistency procedure was $10^{-6} \mathrm{eV}$. We used a $15 \times 15 \times 1$ Monkhorst-Pack k-point grid in the Brillouin zone, with one k-point along the direction perpendicular to the $2 \mathrm{D}$ plane of the heterostructures. To avoid interactions between replicated images, a vacuum buffer of $20 \AA$ was used. All the atomic positions in the systems were fully relaxed until the residual force were less than $0.001 \mathrm{eV} / \AA$ on each atom. Van der Waals (vdW) interactions were treated at the DFT-D3 level. ${ }^{[52]}$

The relative stability of the different heterostructure studied can be estimated by means of their formation energy, $E_{f}$, calculated as:

$$
E_{f}=E_{\text {MoSSe } / \text { GaN }}-E_{\text {Mosse }}-E_{\text {GaN }}
$$

$E_{M o S S e / G a N}, E_{M o S S e}$, and $E_{G a N}$ are the total energy of the MoSSe/GaN heterostructure, and of the single-layer components (MoSSe and $\mathrm{GaN}$ ) optimized in isolation at the same box, respectively. For the systems studied, the sign of $E_{f}$ is negative in general as the systems pack via vdW interactions that are only attractive for a negligible interfacial relaxation. Thus, the more negative $E_{f}$, the larger the energetic favorability of the heterostructure.

The carrier mobility of the two-dimensional heterostructures can be calculated by the phonon-limited formula written as: ${ }^{[53,54]}$

$$
\mu=\left(\mathrm{e} \hbar^{3} C_{2 D}\right) /\left[k_{b} T m^{*} m_{d}\left(E_{i}\right)^{2}\right]
$$

$m^{*}$ is the effective mass ( $m_{e}^{*}$ for electron and $m_{d}^{*}$ for the hole), where $m_{x}$ and $m_{y}$ refer to the effective mass along the $x$ and $y$ direction. $m_{d}^{e / h}$ is the equivalent mass defined as $m_{d}=\sqrt{m_{x} m_{y}}\left(m_{d}^{e}\right.$ for electron and $m_{d}^{h}$ for the hole). $E_{i}(i=e$ for the electron, $i=h$ for the hole $)$ is the deformation potential, calculated as $E_{i}=\Delta E_{i} /\left(\Delta L_{i} / L_{i}\right) . E_{i}$ can be obtained from the band-energy level change $\left(\Delta E_{i}\right)$ induced by a lattice compression or expansion of step $\Delta L_{i} / L_{i}=0.005$ in the transport direction, where the reference energy level is set to the vacuum energy level. Based on this convention, the deformation potentials of one hole in the valence band maximum (VBM) and one electron in the conduction band minimum $(\mathrm{CBM})$ are labelled as $E_{h}$, and $E_{e}$, respectively. 
The elastic moduli of the longitudinal acoustic $C_{2 D}$ in the propagation direction can be calculated as $C_{2 D}=2\left(E-E_{0}\right) /\left[S_{0}\left(\Delta L_{i} / L_{i}\right)^{2}\right]$, where $E$ and $E_{0}$ are the total energy of the compressed/expanded and equilibrium geometries, respectively. $S_{0}$ is the area of two-dimensional material at the equilibrium geometry.

\section{Results and Discussions}

The Janus MoSSe system has a honeycomb hexagonal lattice with three atomic layers as shown in Fig. 1(a). The optimized in plane lattice constant is $a=b=3.215 \AA$, with Mo-S and Mo-Se bond lengths of $2.41 \AA$ and $2.53 \AA$, respectively. Similar to Janus MoSSe, single layer Nitride GaN has also a hexagonal symmetry, as shown in Fig. 1(b). Notably, the lattice constant of GaN is $a=b=3.250 \AA$, only $0.035 \AA$ larger than that of MoSSe. Thus, a rather small ratio of lattice mismatch at about $\sim 1.08 \%$ can be achieved, when MoSSe and GaN form a heterostructure. In the Table 1, there includes other two single layer TMDs as $\mathrm{MoS}_{2}$ and $\mathrm{MoSe}_{2}$ with basic structure parameters, since they will be applied to form heterostructure in the context later.

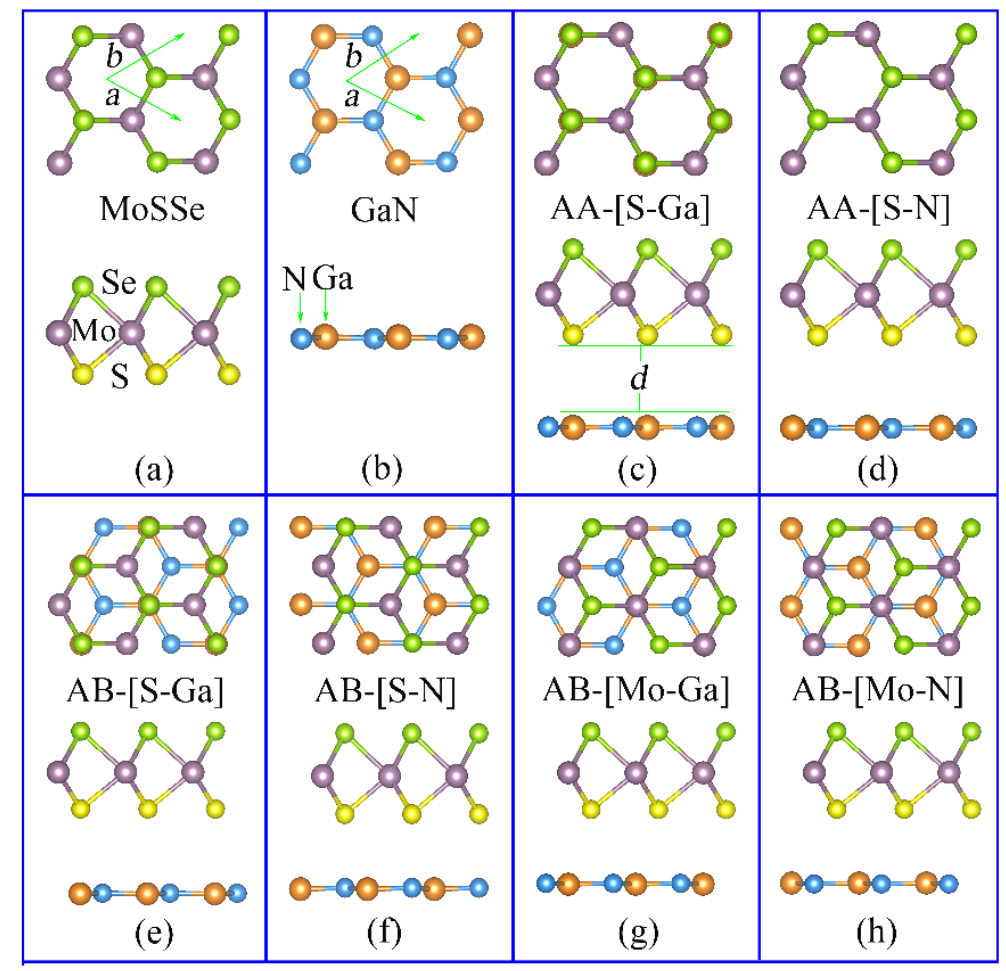

Figure 1. The atomic structure of single layer MoSSe (a), GaN (b), and their AA (c)-(d) and AB stacked (e)-(h) heterostructures in type of SeMoS/GaN. The top and side views are in the upper and lower panels, respectively. The primitive cell is marked by the green arrows. The Mo, S, Se, Ga, and $N$ atoms are shown as light violet, yellow, light green, orange, and gray spheres, respectively. The letter " $d$ " denotes the interlayer distance between the top and bottom layer.

Table 1. The basic structural parameters for the selected single layer TMDs and GaN. The lattice mismatch needed to form the heterostructure and the magnitude of band gap $\left(E_{g}\right)$ are also shown. I and $D$ indicate direct and indirect band gap $\left(E_{g}\right)$, respectively.

\begin{tabular}{|c|c|c|}
\hline Lattice & Length $(\AA)$ & Angle ( $)$ \\
\hline$a=b(\AA)$ & $G a-N$ & $\angle G a N G a$ \\
\hline
\end{tabular}




\begin{tabular}{|c|c|c|c|c|c|c|c|}
\hline System & & Mo-S & Mo-Se & $\angle M O S M O$ & $\angle M o S e M$ & & $E_{g}(e V)$ \\
\hline GaN & 3.250 & \multicolumn{2}{|c|}{1.87} & \multicolumn{2}{|c|}{120} & $0 \%$ & 1.98 (I) \\
\hline MoSSe & 3.215 & 2.41 & 2.53 & 83.73 & 79.09 & $1.08 \%$ & 1.67 (D) \\
\hline $\mathbf{M o S}_{2}$ & 3.152 & 2.403 & & 81.97 & & $3.02 \%$ & 1.78 (D) \\
\hline $\mathrm{MoSe}_{2}$ & 3.287 & & 2.53 & & 80.86 & $1.14 \%$ & 1.54 (D) \\
\hline
\end{tabular}

These results indicate that MoSSe and GaN can form vertical vdW heterostructures (MoSSe/GaN in the following) with minimal in-plane strain. Due to the different faces of MoSSe, two arrangements are possible for such heterostructures, which we refer to as $\mathrm{SMoSe} / \mathrm{GaN}$ and $\mathrm{SeMoS} / \mathrm{GaN}$. In addition, for each arrangement the individual layers can be stacked according to either an $\mathrm{AA}$ or $\mathrm{AB}$ staking pattern, see Fig. 1(c)1(d) and 1(e)-1(h). For the AA-stacked SeMoS/GaN, the $S$ atom can sit above the Ga (denoted as AA-[S-Ga] Fig. 1(c)) or the N atom (denoted as AA-[S-N] in Fig. 1(d)). More combinations become possible for AB-stacking. For the Mo atom of SMoSe/GaN sitting above the hollow-site of the GaN hexagonal lattice, there exist two kinds of possible AB-stacking depending on whether the Se atom sits above a $\mathrm{Ga}$ or $\mathrm{N}$ atom of the underlying GaN lattice. These structures are indicated as AB-[Se-Ga] or AB-[Se-N] as seen in Fig. 1(e) or 1(f). Conversely, for the Se atom of SMoSe/GaN sitting above the hollow-site of the GaN hexagonal lattice, two different AB-stacking are possible depending on whether the Mo atom sits on top of a Ga (AB-[Mo-Ga] in Fig. 1(g)) or N (AB-[Mo-N] in Fig. 1(h)) atom . In the following, the same labelling scheme is used also for the SeMoS/GaN heterostructure with $\mathrm{S}$, not Se, atoms facing the underlying $\mathrm{Ga} / \mathrm{N}$ layer.

Table 2. The calculated formation energy $\left(E_{f}\right)$, interlayer distance $(d)$, and band gap $\left(E_{g}\right)$ for the considered MoSSe/GaN heterostructures in different (AA or AB) stacking. I and $D$ in brackets denote indirect and direct band gap, respectively.

\begin{tabular}{clcccc}
\hline Mode & Stacking & Lattice $(\boldsymbol{A})$ & $\boldsymbol{E}_{\boldsymbol{f}}(\boldsymbol{e} \boldsymbol{V})$ & $\boldsymbol{d}(\boldsymbol{A})$ & $\mathbf{E}_{\mathbf{g}}(\mathbf{e V})$ \\
\hline SeMoS & AA-[S-Ga] & $\mathbf{3 . 2 3 4}$ & $\mathbf{- 0 . 2 6 0}$ & $\mathbf{2 . 9 7 0}$ & $\mathbf{0 . 8 0}(\mathrm{D})$ \\
$/ \mathrm{GaN}$ & AA-[S-N] & 3.233 & -0.166 & 3.497 & 1.20 (D) \\
& AB-[Mo-Ga] & 3.236 & -0.239 & 2.993 & 1.25 (D) \\
& AB-[Mo-N] & 3.234 & -0.230 & 3.053 & 1.15 (D) \\
& AB-[S-Ga] & $\mathbf{3 . 2 3 9}$ & $\mathbf{- 0 . 2 6 6}$ & $\mathbf{2 . 9 4 3}$ & $\mathbf{0 . 8 9}$ (D) \\
& AB-[S-N] & 3.233 & -0.163 & 3.523 & 1.19 (D) \\
SMoSe & AA-[Se-Ga] & $\mathbf{3 . 2 3 7}$ & $\mathbf{- 0 . 2 5 5}$ & $\mathbf{3 . 1 0 7}$ & $\mathbf{1 . 4 5}$ (D) \\
$/$ GaN & AA-[Se-N] & 3.233 & -0.227 & 3.22 & 1.52 (D) \\
& AB-[Ga-Mo] & 3.234 & -0.236 & 3.226 & 1.63 (D) \\
& AB-[Ga-N] & 3.237 & -0.230 & 3.053 & 1.15 (D) \\
& AB-[Se-Ga] & $\mathbf{3 . 2 3 8}$ & $\mathbf{- 0 . 2 4 2}$ & $\mathbf{3 . 1 5 4}$ & $\mathbf{1 . 3 8}$ (D) \\
& AB-[Se-N] & 3.237 & -0.167 & 3.592 & 1.62 (D) \\
SMoS & AA-[S-Ga] & $\mathbf{3 . 2 1 0}$ & $\mathbf{- 0 . 2 1 2}$ & $\mathbf{2 . 9 8 8}$ & $\mathbf{0 . 7 7}$ (D) \\
\hline
\end{tabular}




\begin{tabular}{|c|c|c|c|c|c|}
\hline$/ \mathrm{GaN}$ & $\mathrm{AA}-[\mathrm{S}-\mathrm{N}]$ & 3.207 & -0.121 & 3.036 & 1.13 (D) \\
\hline SMoS & $\mathrm{AB}-[\mathrm{Mo}-\mathrm{Ga}]$ & 3.208 & -0.19 & 3.036 & 1.22 (D) \\
\hline \multirow[t]{3}{*}{$/ \mathrm{GaN}$} & AB-[Mo-N] & 3.206 & -0.184 & 3.103 & 1.13 (D) \\
\hline & AB-[S-Ga] & 3.212 & -0.209 & 2.967 & 0.84 (D) \\
\hline & $\mathrm{AB}-[\mathrm{S}-\mathrm{N}]$ & 3.206 & -0.118 & 3.526 & 1.12 (D) \\
\hline SeMoSe & AA-[Se-Ga] & 3.265 & -0.265 & 3.106 & 1.47 (D) \\
\hline$/ \mathrm{GaN}$ & AA-[Se-N] & 3.261 & -0.172 & 3.573 & 1.56 (D) \\
\hline SeMoSe & $\mathrm{AB}-[\mathrm{Mo}-\mathrm{Ga}]$ & 3.263 & -0.237 & 3.119 & 1.53 (D) \\
\hline \multirow[t]{3}{*}{$/ \mathrm{GaN}$} & AB-[Mo-N] & 3.261 & -0.241 & 3.169 & 1.59 (D) \\
\hline & AB-[Se-Ga] & 3.266 & -0.255 & 3.105 & 1.41 (D) \\
\hline & AB-[Se-N] & 3.261 & -0.170 & 3.609 & $1.54(\mathrm{I})$ \\
\hline
\end{tabular}

Table 2 reports the calculated formation energy, lattice constant, interlayer distance, and band gap of the different $\mathrm{MoSSe} / \mathrm{GaN}$ heterostructures studied. The simulations indicate that AB-[S-Ga] stacking results in the lowest $E_{f}$, of $-0.266 \mathrm{eV}$ in $\mathrm{SeMoS} / \mathrm{GaN}$, indicating this arrangement as the energetically favored. AA-[Se-Ga] stacking of the $\mathrm{SMoSe} / \mathrm{GaN}$ heterostructure yields the second lowest $E_{f}$. Substitution of the upper MoSSe layer of the heterostructure with $\mathrm{MoS}_{2}(\mathrm{SMoS})$ or $\mathrm{MoSe}_{2}(\mathrm{SeMoSe})$ layers result in the AA-[S-Ga] $\left(\mathrm{MoS}_{2} / \mathrm{GaN}\right)$ and AA-[Se-Ga] $\left(\mathrm{MoSe}_{2} / \mathrm{GaN}\right)$ stacking being energetically favored with $E_{f}$ of $-0.212 \mathrm{eV}$ and $-0.265 \mathrm{eV}$, respectively. These results deviate from what previously calculated for heterostructures in graphene, for which AB-stacking is energetically favored. ${ }^{[55]}$ Furthermore, we can also find that different stackings own different interlayer distance. This may be related to the possible atomic position and environment. Considering AA-[S-Ga] and AA-[S-N], the metal atom Ga is attractive to nonmetal $\mathrm{S}$ in AA-[S-Ga], while both nonmetal atom $\mathrm{N}$ and $\mathrm{S}$ in AA-[S$\mathrm{N}]$ is repulsive to each other, leading to a larger interlayer distance of $3.5 \AA$ in AA-[S$\mathrm{N}]$. The results in Table 2 indicates also that $E_{f}$ for the AB-[S/Se-Ga] stacking in general is comparable (to within $0.013 \mathrm{eV}$ ) to the $\mathrm{AA}-[\mathrm{S} / \mathrm{Se}-\mathrm{Ga}]$ one, suggesting these patterns to be energetically competitive and potentially accessible by experiments.

The lower formation energy is related to the different electron structure and interlayer distance of the heterostructure. As for SMoS/GaN, S atoms for AA-[S-Ga] in negative valence attracts the $\mathrm{Ga}$ atoms in positive valence with lower interlayer distance, resulting in stabilization of the heterostructure. Whereas the $\mathrm{S}$ atoms repels the $\mathrm{N}$ atoms in AA-[S-N] or other AB-stacking configurations and hence destabilizes the structures. Further, owing to the stronger oxidation of $\mathrm{S}$ atom than that of Se atom, the AA-[S-Ga] in $\mathrm{SeMoS} / \mathrm{GaN}$ is about $-0.01 \mathrm{eV}$ lower than AA-[Se-Ga] in SMoSe/GaN. Except for the formation energy, the thermal stability of these heterostructures are also checked by first-principles molecular dynamics (FPMD). Here, we choose MoSSe/GaN heterostructure as a typical example. It is interesting to find that the structure remains unbroken for 2 ps FPMD at $300 \mathrm{~K}$ as shown in SI-Fig. 1, suggesting the structure is thermal stable. Therefore, the results show that $\mathrm{AB}-[\mathrm{S}-\mathrm{Ga}]$ is more stable in $\mathrm{SeMoS} / \mathrm{GaN}$, while AA-[S/Se-Ga] is always more stable than other stackings for other heterostructures.

Besides the structural stability and formation energy, we analyzed also the electronic properties of the heterostructures studies. The corresponding results in Table 2 show 
that all the heterostructures studied are semiconductors regardless of the stacking type and relative atomic positions. The calculated band gap varies from $0.767 \mathrm{eV}$ (SMoS/GaN, AA-[S-Ga]) to $1.631 \mathrm{eV}(\mathrm{SMoSe} / \mathrm{GaN}, \mathrm{AB}-[\mathrm{Ga}-\mathrm{Mo}])$, which is located in the near visible-infrared region. Given the known underestimation errors the used PBE-GGA functional in calculating band-gap, these results suggest that visible light absorption for these systems may be possible. As also shown in Table 2, we find most of the heterostructures studied to have a direct band gap, in contrast with the indirect band gap for pristine GaN. Therefore, the simulations suggest that direct tuning of the electronic properties and, inevitably, light absorption of the heterostructures can be achieved by controlling the stacking geometry.

To further analyze the electronic properties of the lowest $E_{f}$ heterostructures, we show their calculated band structures in Fig. 2. For all the systems, both the VBM and CBM are located at the $\mathrm{K}$ point, suggesting that all these heterostructures are direct semiconductors. By changing the position of the $\mathrm{S}$ and $\mathrm{Se}$ atoms with respect to the underlying GaN layer, the band gap can vary from 0.77 to $1.47 \mathrm{eV}$. In order to check the accuracy of PBE result, HSE hybrid functional was also considered. Here, we considered $\mathrm{AB}-[\mathrm{S}-\mathrm{Ga}]$ and $\mathrm{AA}-[\mathrm{S}-\mathrm{Ga}]$ of $\mathrm{MoSSe} / \mathrm{GaN}$ as typical examples, and the corresponding band structures are shown in supporting information SI-Fig. 2. It can be found that the band gaps are about $0.6 \mathrm{eV}$ larger relative to the ones with PBE, but the tendency of the calculated band gaps with HSE is similar to the one with PBE.

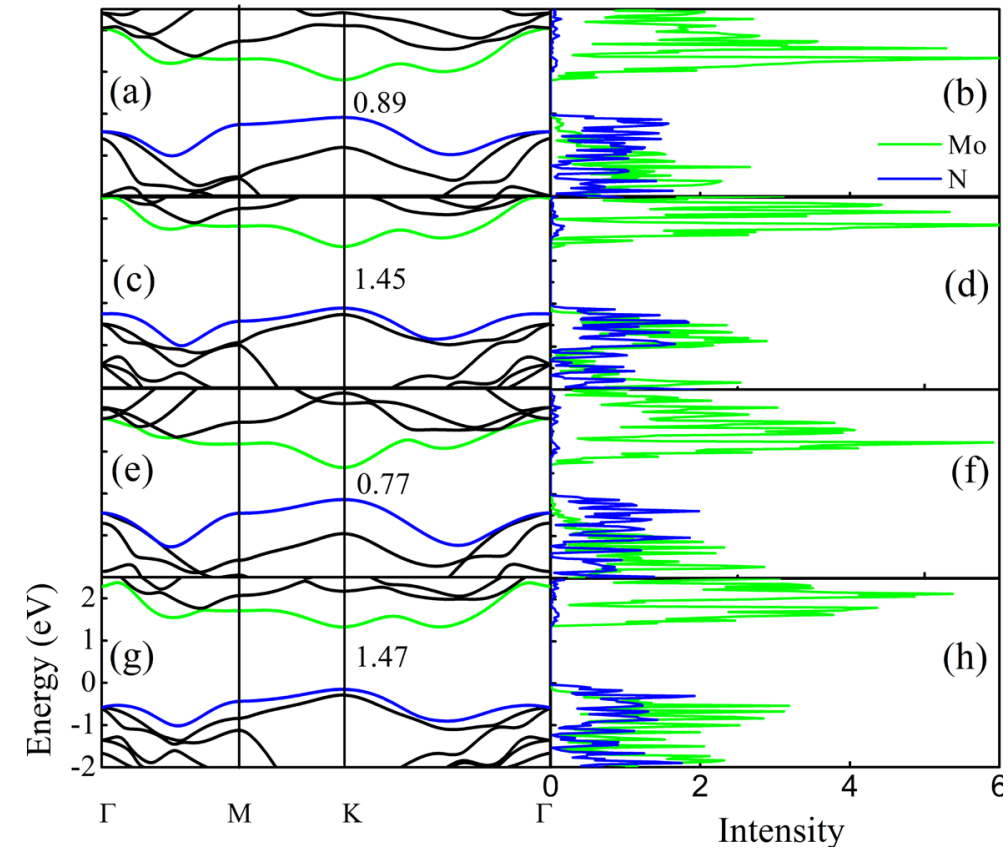

Figure 2. The calculated electron band structure and atom-projected density of states (PDOS) for the energy favored stacking of the MoSSe/GaN, MoS $/ \mathrm{GaN}$ and $\mathrm{MoSe}_{2} / \mathrm{GaN}$ heterostructure. (a-b) AB-[S-Ga] stacked SeMoS/GaN, (c-d) AA-[Se-Ga] stacked SMoSe/GaN, (d-e) AA-[S-Ga] stacked MoS $/ G a N$, and (e-f) AA-[Se-Ga] stacked $\mathrm{MoSe}_{2} / \mathrm{GaN}$. The lowest and highest energy bands in the CB and VB are shown in green and blue, respectively. The Mo and $N$ resolved PDOS are shown in green and blue, respectively.

To investigate the atomic contributions to $\mathrm{VB}$ and $\mathrm{CB}$, Fig. 2 reports also the 
calculated electron, atom-projected density of states (PDOS). For clarity, only the main contributions to the VBM and CBM are provided. The results indicate that, for all the systems, the main contribution to the VBM stem from the $\mathrm{N}_{2 p}$ orbitals (blue line) of GaN. Conversely, the main contributions to the CBM are due to the Mo3d orbitals (green line) of MoSSe, $\mathrm{MoS}_{2}$ and $\mathrm{MoSe}_{2}$. Therefore, the CBM and VBM are distributed on different side of the heterostructure. This result can be straightforwardly visualized by the band-decomposed charge densities for the VBM and CBM, shown for SMoSe/GaN as model system in Fig. 3. Evidently, whereas the CBM charge density for AA-[Se-Ga] is mostly localized on the Mo atoms of the SMoSe layer, the VBM charge density is largest on the $\mathrm{N}$ atoms for the $\mathrm{GaN}$ layer. This intriguing property suggests that there is an intrinsic electronic field in the $\mathrm{SMoSe} / \mathrm{GaN}$ heterostructure, which should be highly beneficial to separate photoinduced carriers.

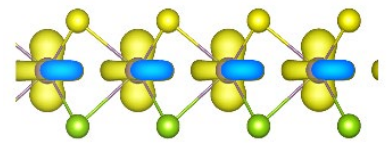

(a)

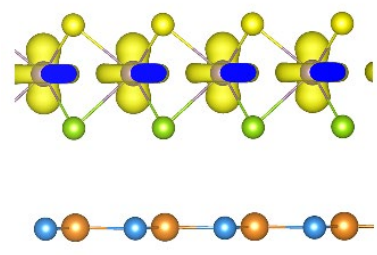

(c)

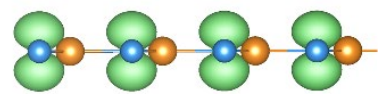

(b)

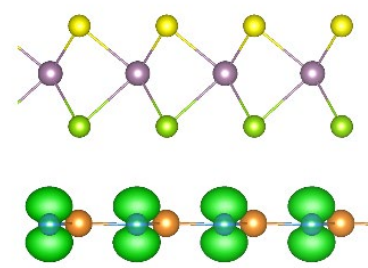

(d)

Figure 3. (Color online) (a)-(b) Side views of the density-distribution of the CBM in SMoSe (a) and of the VBM in GaN (b). (c)-(d) The side view of density-distribution of the the CBM and the VBM for the MoSSe bilayer. The isovalue for the contour plot is 0.01 e bohr $r^{-3}$.

The calculated dependence of the heterostructures electronic properties on the stacking geometry prompts for investigation of its role for carrier mobility too. We start by benchmarking the accuracy of our approach to electron effective mass and mobility for single layer $\mathrm{MoS}_{2}$. We calculate an effective mass and carrier mobility of the electron in the CBM along X-K of $0.50 \mathrm{~m}_{0}$ and $145.5 \mathrm{~cm}^{2} \mathrm{~V}^{-1} \mathrm{~s}^{-1}$, close to previously experimental result of $0.49 \mathrm{~m}_{0}$ and $200 \mathrm{~cm}^{2} \mathrm{~V}^{-1} \mathrm{~s}^{-1} \cdot{ }^{19]}$ Literally, the calculated result is agree to the previous work, suggesting it is relatively reliable.

Table 3 reports the calculated carriers $m^{*}, m_{d}, E_{i}, C_{2 D}$, and $\mu$ for $\mathrm{MoSSe} / \mathrm{GaN}$, as well as $\mathrm{MoS}_{2} / \mathrm{GaN}$ and $\mathrm{MoSe}_{2} / \mathrm{GaN}$ in different stacking, together with results for single layer TMDs and GaN for comparison. For this analysis, we focus on the heterostructures with the lowest and second lowest $E_{f}$. Starting with MoSSe/GaN, the $\mathrm{CBM} \mu_{e}$ of AB-[S-Ga] stacked SeMoS/GaN along the X-K path is about 263.28 $\mathrm{cm}^{2} \mathrm{~V}^{-1} \mathrm{~s}^{-1}$, which is nearly three times larger than for single layer MoSSe $\left(97.83 \mathrm{~cm}^{2} \mathrm{~V}^{-}\right.$ $\left.{ }^{1} \mathrm{~s}^{-1}\right)$. Conversely, the VBM $\mu_{h}$ along the X-K path is about $3480.43 \mathrm{~cm}^{2} \mathrm{~V}^{-1} \mathrm{~s}^{-1}$, slightly smaller than for single layer GaN $\left(3785.03 \mathrm{~cm}^{2} \mathrm{~V}^{-1} \mathrm{~s}^{-1}\right)$. Qualitatively different results are computed for the other arrangements of the $\mathrm{SMoSe} / \mathrm{GaN}$ heterostructure. The calculated $\mu_{e}$ and $\mu_{h}$ of AA-[Se-Ga] are increased to 281.28 and $3951.21 \mathrm{~cm}^{2} \mathrm{~V}^{-1} \mathrm{~s}^{-1}$, 
respectively. These values are larger than for the isolated MoSSe and GaN layers. Therefore, the simulations indicate that the carrier mobility of MoSSe/GaN heterostructures can be substantially, up to nearly three times, larger than for the individual components.

Analysis of the carrier mobility can be extended by considering the role of $\mathrm{S}$ or $\mathrm{Se}$ exposure to $\mathrm{GaN}$ for calculated results. As the Se atom in MoSSe is substituted by $\mathrm{S}$ to form the $\mathrm{MoS}_{2} / \mathrm{GaN}$ heterostructure, the $\mu_{e}$ of AA-[S-Ga] decreases to $143.62 \mathrm{~cm}^{2} \mathrm{~V}^{-}$ ${ }^{1} \mathrm{~s}^{-1}$. Also, the $\mu_{h}$ of AA-[S-Ga] stacked $\mathrm{MoS}_{2} / \mathrm{GaN}$ decreases slightly (by $40.3 \mathrm{~cm}^{2} \mathrm{~V}^{-}$ ${ }^{1} \mathrm{~s}^{-1}$ ) relative to the AA-[Se-GaN] stacking of MoSSe/GaN. In turn, as the $\mathrm{S}$ atom of MoSSe is changed into a Se atom to form $\mathrm{MoSe}_{2} / \mathrm{GaN}$ heterostructure, the $\mu_{e}$ becomes $258.25 \mathrm{~cm}^{2} \mathrm{~V}^{-1} \mathrm{~s}^{-1}$ similar to the value for $\mathrm{SMoSe} / \mathrm{GaN}$. In contrast, the $\mu_{h}$ is strongly decreased by $3000 \mathrm{~cm}^{2} \mathrm{~V}^{-1} \mathrm{~s}^{-1}$. Thus, depending on the heterostructure composition, the $\mu_{e}$ can be tuned from $143.62 \mathrm{~cm}^{2} \mathrm{~V}^{-1} \mathrm{~s}^{-1}$ to $281.28 \mathrm{~cm}^{2} \mathrm{~V}^{-1} \mathrm{~s}^{-1}$ in the $\mathrm{CBM}$, and the $\mu_{h}$ from $791.34 \mathrm{~cm}^{2} \mathrm{~V}^{-1} \mathrm{~s}^{-1}$ to $3951.21 \mathrm{~cm}^{2} \mathrm{~V}^{-1} \mathrm{~s}^{-1}$ in the VBM.

The simulations reveal an important role also of the heterostructure stacking for its carrier mobility. For instance, the $\mu_{e}$ and $\mu_{h}$ of AA-[S-Ga] stacked SeMoS/GaN are about 13.82 and $1002 \mathrm{~cm}^{2} \mathrm{~V}^{-1} \mathrm{~s}^{-1}$ smaller than for the analogous AB-[S-Ga] stacked system. This trend is different from what shown by the other systems studied, for which AA stacking is found to increase carrier mobility. Taking SMoSe/GaN as an example, the $\mu_{e}$ and $\mu_{h}$ of AA-[Se-Ga] is increased by 8.38 and $259.06 \mathrm{~cm}^{2} \mathrm{~V}^{-1} \mathrm{~s}^{-1}$ compared to $\mathrm{AB}-[\mathrm{Se}-\mathrm{Ga}]$. Thus, we find that, depending on the $\mathrm{AB}$ or AA stacking, carrier mobility can be tuned by $19 \sim 123 \mathrm{~cm}^{2} \mathrm{~V}^{-1} \mathrm{~s}^{-1}$ for electrons, and by $259 \sim 1002 \mathrm{~cm}^{2} \mathrm{~V}^{-1} \mathrm{~s}^{-1}$ for holes. Comparing these results with the substantially larger compositional changes analyzed above, we are to conclude that carrier mobility in the considered heterostructures are substantially more sensitive to the occurrence of $\mathrm{S} / \mathrm{GaN}$ or $\mathrm{Se} / \mathrm{GaN}$ interfaces rather than AA or AB stacking.

Table 3. The calculated carrier mobility at $300 \mathrm{~K}$ for the (lowest and second lowest $E_{f}$ ) $\mathrm{MoSSe} / \mathrm{GaN}, \mathrm{MoS}_{2} / \mathrm{GaN}$, and $\mathrm{MoSe}_{2} /$ GaN heterostructures. The results for single layer Janus MoSSe, MoS, $\mathrm{MoSe}_{2}$, and GaN are also provided for comparison. $m_{e}^{*} / m_{h}^{*}$ : the effective electron/hole mass, $m_{d}^{e} / m_{d}^{h}$ : the effective equivalent electron/hole mass, $E_{e} / E_{h}$ : the electron/hole deformation potential, $C_{2 D}$ : the elastic moduli, and $\mu_{e} / \mu_{h}$ : the electron/hole carrier mobility at CBM and VBM. It should be mentioned that only lowest $E_{f}$ and second lowest $E_{f}$ cases are considered.

\begin{tabular}{|c|c|c|c|c|c|c|c|c|c|c|}
\hline & System & $m_{e}^{*}$ & $m_{d}^{e}$ & $m_{h}^{*}$ & $m_{d}^{h}$ & $E_{e}$ & $E_{h}$ & $C_{2 D}$ & $\mu_{e}$ & $\mu_{h}$ \\
\hline & GaN & 0.22 & 0.72 & 1.27 & 0.89 & 10.6 & 0.84 & 140.2 & 171.10 & 3785.03 \\
\hline & $\mathbf{M o S}_{2}$ & 0.50 & 0.62 & 0.60 & 1.57 & 8.22 & 2.30 & 141.15 & 145.50 & 605.44 \\
\hline & MoSSe & 0.56 & 0.62 & 0.68 & 2.61 & 8.46 & 2.83 & 115.39 & 97.83 & 173.65 \\
\hline & $\mathrm{MoSe}_{2}$ & 0.53 & 0.5 & 0.64 & 2.42 & 6.94 & 2.16 & 204.12 & 263.15 & 600.77 \\
\hline SeMoS & $\mathrm{AA}-[\mathrm{S}-\mathrm{Ga}]$ & 0.53 & 0.66 & 1.49 & 1.06 & 8.0 & 1.20 & 264.10 & 249.46 & 2478.36 \\
\hline$/ \mathbf{G a N}$ & AB-[S-Ga] & 0.55 & 0.65 & 1.80 & 1.26 & 7.66 & 0.84 & 258.98 & 263.28 & 3480.43 \\
\hline SMoSe & AA-[Se-Ga] & 0.54 & 0.7 & 1.23 & 1.45 & 7.47 & 0.91 & 275.5 & 281.28 & 3951.21 \\
\hline$/ \mathrm{GaN}$ & AB-[Se-Ga] & 0.52 & 0.70 & 1.82 & 0.84 & 7.62 & 1.00 & 266.86 & 272.90 & 3692.15 \\
\hline $\mathbf{M o S}_{2} /$ & AA-[S-Ga] & 0.44 & 1.47 & 1.27 & 1.17 & 8.05 & 1.02 & 273.87 & 143.62 & 3910.91 \\
\hline
\end{tabular}




\begin{tabular}{ccccccccccc}
\hline GaN & AB-[S-Ga] & 0.44 & 1.53 & 1.49 & 1.07 & 6.24 & 2.83 & 277.79 & 266.93 & 3050.20 \\
MoSe $_{2} /$ & AA-[Se-Ga] & $\mathbf{0 . 7 3}$ & $\mathbf{0 . 6 9}$ & $\mathbf{1 . 3 9}$ & $\mathbf{1 . 1 4}$ & $\mathbf{6 . 6 6}$ & $\mathbf{2 . 1 6}$ & $\mathbf{2 7 1 . 9 1}$ & $\mathbf{2 5 8 . 2 5}$ & $\mathbf{7 9 1 . 3 4}$ \\
GaN & AB-[Se-Ga] & 0.61 & 0.68 & 1.00 & 0.99 & 8.41 & 1.81 & 265.58 & 191.53 & 1739.03 \\
\hline
\end{tabular}

In order to identify the diverse carrier mobility in heterostructures studied, we systemically calculated carriers effective and equivalent mass, deformation potential, and elastic moduli for the heterostructures as well as their individual component as shown in Table 3. Identification of the crucial factor(s) behind the carrier mobility is essential for definition of synthetic strategies to enhance it. Taking AA-[Se-Ga] stacked $\mathrm{SMoSe} / \mathrm{GaN}$ as case study, the $\mu_{e}$ is about 2 times that of MoSSe, and the $\mu_{h}$ is close to isolated $\mathrm{GaN}$ layer. However, the ratio of $m_{e}^{*}, m_{d}^{e}, E_{e}$, and $C_{2 D}$ between AA-[Se$\mathrm{Ga}]$ and MoSSe is about $0.98,1.13,0.88$, and 2.4, while the ratio of $m_{h}^{*}, m_{d}^{h}, E_{h}$, and $C_{2 D}$ between AA-[Se-Ga] and $\mathrm{GaN}$ is about $0.97,1.63,1.08$, and 1.96. According to Eq. (2), $m^{*}, m_{d}$, and $E_{i}$ are inversely proportional, while the $C_{2 D}$ is linearly proportional to the $\mu$. Thus, it can be deduced that the $\mu_{e}$ is strongly relative to the $C_{2 D}$, whereas the $\mu_{h}$ is also related to $m_{d}$. The case for AA-[S-Ga] of $\mathrm{MoS}_{2} / \mathrm{GaN}$ is a little different, where the $\mu_{e}$ is close to that of $\mathrm{MoS}_{2}$, and the $\mu_{h}$ is a little larger than isolated GaN layer. The ratio of $m_{e}^{*}, m_{d}^{e}, E_{e}$, and $C_{2 D}$ between AA-[S-Ga] and $\mathrm{MoS}_{2}$ is about $0.88,2.37,0.98$, and 1.94, while the ratio of $m_{h}^{*}, m_{d}^{h}, E_{h}$, and $C_{2 D}$ between AA-[S-Ga] and GaN is about 1.00, 1.31, 1.21, and 1.94. So, it can be found that the $\mu_{e}$ is strongly affected by $m_{d}^{e}$, whereas the $\mu_{h}$ is much sensitive to $E_{h}$. As a result, we can find that the carrier mobility is strongly affected by larger equivalent mass and elastic moduli, meanwhile, the hole carrier mobility is also related to deformation potential. As for the larger equivalent mass, it can clearly see from the extremely flat character of the CMB along the $\Gamma-\mathrm{M}$ path (Fig. 2). Considering elastic moduli, the larger elastic moduli in heterostructure should origin from the thicker thickness in heterostructure compared with those of its single component.

Then, it is interesting to know the reason why electron and hole behaves markedly differently. As for SMoSe/GaN, the electron mobility of AA-[Se-Ga] is $281.28 \mathrm{~cm}^{2} \mathrm{~V}^{-}$ ${ }^{1} \mathrm{~S}^{-1}$, while the hole mobility is $3951.21 \mathrm{~cm}^{2} \mathrm{~V}^{-1} \mathrm{~s}^{-1}$ about 14 times larger than for the electron. The ratio of effective mass, equivalent mass, deformation potential, and elastic moduli between hole and electron are about 2.23, 2.1, 0.12, and 1.0. Accordingly, we are to infer that the deformation potential should be the dominant factor. The different deformation potential can be seen from the different bonding characteristic of valence band and conduction band. For the electron in CBM, the charge distribution is mainly localized in the $x-y$ plane in Mo3d orbitals (see Fig. 3(c)), which is larger than the distribution of VBM with the electron localized only at $\mathrm{N}_{2 p}$ orbitals (see in Fig. 3(d)). As structural deformation occurs, it will generate stronger electrostatic interaction in CBM than that in VBM, corresponding to larger $E_{e}$ than $E_{h}$. Therefore, smaller deformation potential contributes to larger $\mu_{h}$ for the heterostructure.

As discussed above, the electron and hole carrier mobility can also be influenced by stacking of the heterostructure. As for stacking effect, the result indicates that the carrier mobility of second lowest $E_{f}$ structure is always smaller than for the lowest $E_{f}$ stacking. This difference should origin from a slight larger equivalent mass and deformation potential of the distinct atoms. 


\section{Conclusion}

In summary, the structural, electronic and carrier mobility properties of $\mathrm{MoSSe} / \mathrm{GaN}$ heterostructures were systematically investigated by the first-principles calculations. It shows that Janus MoSSe/GaN heterostructure has a relatively high carrier mobility of $281.28 \mathrm{~cm}^{2} \mathrm{~V}^{-1} \mathrm{~s}^{-1}$ for electron and $3951.2 \mathrm{~cm}^{2} \mathrm{~V}^{-1} \mathrm{~s}^{-1}$ for hole. Except the intrinsic electric field of Janus MoSSe, the carrier mobility can be further regulated through the different stacking strategies and Janus atomic structure of MoSSe. The results further indicate that the superior carrier mobility of Janus MoSSe/GaN heterostructure are affected by the equivalent mass and elastic moduli. These results showed that the Janus $\mathrm{MoSSe} / \mathrm{GaN}$ heterostructure has a potential to be used as electronic device for their carrier mobility.

\section{Acknowledgements}

This work was supported by the Science Challenge Project (TZ2018004), the National Natural Science Foundation of China (Nos. 51572016, U1530401, 11747167, 11804090, 51472209, 11774298, U1401241, and 21503012), the Natural Science Foundation of Hunan Province, China (Grant No. 17C0626, and 2019JJ50148), and the Scientific Research Fund of University (No. E517558). This research was also supported by a Tianhe-2JK computing time award at the Beijing Computational Science Research Center (CSRC). L.M.L. and G. T. acknowledge also support by the Royal Society Newton Advanced Fellowship scheme (grant No. NAF\R1\180242).

\section{References}

[1]Y. Gao, T. Cao, F. Cellini, C. Berger, W. A. de Heer, E. Tosatti, E. Riedo, and A. Bongiorno, Nat Nanotechnol 13, 133 (2018).

[2]C. Tan, et al., Chem. Rev. 117, 6225 (2017).

[3]W.-J. Yin, B. Wen, C. Zhou, A. Selloni, and L.-M. Liu, Surface Science Reports 73, 58 (2018).

[4]M. Wang, Y. Pang, D. Y. Liu, S. H. Zheng, and Q. L. Song, Computational Materials Science 146, 240 (2018).

[5]A. K. Geim, Science 324, 1530 (2009).

[6]K. S. Novoselov, A. K. Geim, S. V. Morozov, D. Jiang, Y. Zhang, S. V. Dubonos, I. V. Grigorieva, and A. A. Firsov, Science 306, 666 (2004).

[7]C. Lin, D. Shin, and A. A. Demkov, Journal of Applied Physics 117, 225703 (2015).

[8]Z.-K. Han, Y.-Z. Yang, B. Zhu, M. V. Ganduglia-Pirovano, and Y. Gao, Physical Review Materials 2 , 035802 (2018).

[9]T. Umebayashi, T. Yamaki, H. Itoh, and K. Asai, Journal of Physics and Chemistry of Solids 63, 1909 (2002).

[10]H. C. Yang, Y. Xie, J. Hou, A. K. Cheetham, V. Chen, and S. B. Darling, Adv Mater 30, e1801495 (2018).

[11]Y.-N. Z. Hui Zhang, Hao Liu and Li-Min Liu, J. Mater. Chem. A 2, 15389 (2014).

[12]Y. Gong, et al., Nature Nanotechnology 13, 294 (2018).

[13]M. Chhowalla, H. S. Shin, G. Eda, L.-J. Li, K. P. Loh, and H. Zhang, Nat Chem 5, 263 (2013).

[14]C. Ataca, H. Şahin, and S. Ciraci, The Journal of Physical Chemistry C 116, 8983 (2012).

[15]Q. Xiang, J. Yu, and M. Jaroniec, Journal of the American Chemical Society 134, 6575 (2012).

[16]B. Radisavljevic and A. Kis, Nature Materials 12, 815 (2013). 
[17]W. S. Yun, S. W. Han, S. C. Hong, I. G. Kim, and J. D. Lee, Physical Review B 85, 033305, (2012). [18]S. Fathipour, et al., Applied Physics Letters 105, 192101 (2014).

[19]RadisavljevicB, RadenovicA, BrivioJ, GiacomettiV, and KisA, Nat Nano 6, 147 (2011).

[20]Y. Guo, Q. Wu, Y. Li, N. Lu, K. Mao, Y. Bai, J. Zhao, J. Wang, and X. C. Zeng, Nanoscale Horizons 4, 223 (2019).

[21]Y. Guo, L. Ma, K. Mao, M. Ju, Y. Bai, J. Zhao, and X. C. Zeng, Nanoscale Horizons 4, 592 (2019).

[22]G. Mattioli, F. Filippone, P. Alippi, and A. Amore Bonapasta, Physical Review B 78, 241201(R) (2008).

[23]S. Najmaei, et al., Nature Materials 12, 754 (2013).

[24]X. Ma, X. Wu, H. Wang, and Y. Wang, Journal of Materials Chemistry A 6, 2295 (2018).

[25]R. Chaurasiya and A. Dixit, Applied Surface Science 490, 204 (2019).

[26]Y. Cai, G. Zhang, and Y. W. Zhang, Journal of the American Chemical Society 136, 6269 (2014).

[27]A.-Y. Lu, et al., Nature Nanotechnology 12, 744 (2017).

[28]J. Zhang, et al., ACS nano 11, 8192 (2017).

[29]R. Peng, Y. Ma, S. Zhang, B. Huang, and Y. Dai, The Journal of Physical Chemistry Letters 9, 3612 (2018).

[30]W.-J. Yin, B. Wen, G.-Z. Nie, X.-L. Wei, and L.-M. Liu, Journal of Materials Chemistry C 6, 1693 (2018).

[31]H. C. Yang, J. Hou, V. Chen, and Z. K. Xu, Angew Chem Int Ed Engl 55, 13398 (2016).

[32]Y. Guo, S. Zhou, Y. Bai, and J. Zhao, Applied Physics Letters 110, 163102 (2017).

[33]L. Dong, J. Lou, and V. B. Shenoy, ACS nano 11, 8242 (2017).

[34]H. L. Wang, L. S. Zhang, Z. G. Chen, J. Q. Hu, S. J. Li, Z. H. Wang, J. S. Liu, and X. C. Wang, Chem. Soc. Rev. 43, 5234 (2014).

[35]Y.-m. Ding, et al., Nanoscale 9, 14682 (2017).

[36]F.-B. Zheng, L. Zhang, J. Zhang, P.-j. Wang, and C.-W. Zhang, Physical Chemistry Chemical Physics 22, 5163 (2020).

[37]Yongsheng Yao, Juexian Cao, Wenjin Yin, Liwen Yang, and Xiaolin Wei, J. Phys. D: Appl. Phys. 53, $5,(2020)$.

[38]W. Li, Z. Lin, and G. Yang, Nanoscale 9, 18290 (2017).

[39]X. Sun, H. Deng, W. Zhu, Z. Yu, C. Wu, and Y. Xie, Angew Chem Int Ed Engl 55, 1704 (2016).

[40]Z. Wei, F. F. Liang, Y. F. Liu, W. J. Luo, J. Wang, W. Q. Yao, and Y. F. Zhu, Appl. Catal. B-Environ. 201, 600 (2017).

[41]W. Yin, B. Wen, Q. Ge, D. Zou, Y. Xu, M. Liu, X. Wei, M. Chen, and X. Fan, Progress in Natural Science: Materials International 29, 335 (2019).

[42]W. Yin, B. Wen, Q. Ge, X. Wei, G. Teobaldi, and L. Liu, Progress in Natural Science: Materials International 30, 128 (2020).

[43]M. L. Sun, J. P. Chou, Q. Q. Ren, Y. M. Zhao, J. Yu, and W. C. Tang, Appl. Phys. Lett. 110, 173105 (2017).

[44]Z. L. Lu, D. Y. Zhi, G. Jing, C. R. Ze, X. Y. Zong, and L. Wei, Mater. Lett. 213, 387 (2018).

[45]Z. Y. Al Balushi, et al., Nature materials 15, 1166 (2016).

[46]Y. Zhao, H. Wang, H. Zhou, and T. Lin, Small 13 (2017).

[47]X. Li, Z. Li, and J. Yang, Physical review letters 112, 018301 (2014).

[48]G. Kresse and J. Hafner, Physical Review B 47, 558 (1993).

[49]G. K. a. J. Furthmuler, Physical Review B 54, 11169 (1996). 
[50]J. P. Perdew, K. Burke, and M. Ernzerhof, Physical Review Letters 77, 3865 (1996).

[51]J. P. Perdew, K. Burke, and M. Ernzerhof, Physical Review Letters 78, 1396 (1997).

[52]S. Grimme, J. Antony, S. Ehrlich, and H. Krieg, J. Chem. Phys. 132, 154104(1) (2010).

[53]X.-B. Li, P. Guo, Y.-N. Zhang, R.-F. Peng, H. Zhang, and L.-M. Liu, Journal of Materials Chemistry C 3, 6284 (2015).

[54]S. Bruzzone and G. Fiori, Applied Physics Letters 99, 222108 (2011).

[55]C. L. Lu, C. P. Chang, Y. C. Huang, R. B. Chen, and M. L. Lin, Physical Review B 73, 144427 (2006). 